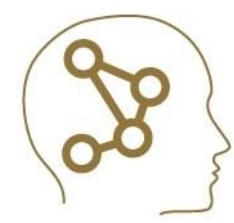

\title{
Computational Framework of Goal Directed Voluntary Motion Generation and Control Loop in Humanoid Robots
}

\author{
Evren Daglarli (i) \\ Istanbul Technical University, Computer and Informatics Engineering Faculty, Istanbul, Turkey. (e-mail: evren.daglarli@itu.edu.tr).
}

\section{ARTICLE INFO}

Received: Feb.,09.2021

Revised: March.,19.2021

Accepted: May.,21.2021

\section{Keywords:}

Motion generation

Control loop

Motion correction

Motion disorders

Humanoids

Corresponding author: Evren

Daglarli

$\triangle$ evren.daglarli@itu.edu.tr

용 +902122857535

ISSN: 2548-0650

DOI: https://doi.org/10.52876/jcs.935773

\begin{abstract}
In this paper, it is aimed to construct a computational framework related to bio-inspired motion generation and control systems for humanoid robots. To acquire natural motion patterns in humanoid robots, behaviors observed from biological motor systems in humans and other mammals should be analyzed in detail. Computational mechanisms are mainly placed on the biophysical plausible neural structures embodied in different dynamics. The main components of the system are composed of the limbic system, neocortex, cerebellum, brainstem, and spinal cord modules. Internal dynamics of these modules include a nonlinear estimator (e.g. chaotic attractor), memory formation, learning (neural plasticity) procedure. While the proposed novel neurocognitive framework is performing goal-directed voluntary motion generation and control tasks, also it estimates the amount of motion errors and computes motion correction signals. By this study, some motion-based central nervous system lesions (e.g. epilepsy, Parkinson, etc.) can be computationally modeled so that impairments of motor control commands are detected. Thus motion disorders can be reconstructed not only in humanoid robots but also in humans via some locomotion equipment.
\end{abstract}

\section{INTRODUCTION}

$\mathrm{T}$ HE motion-based behavioral activity is very crucial for soft robotic systems which are diverse time-varying, nonrigid flexible dynamical systems [1]. Recently, these systems have gained the opportunity to interact more with their environment or people in many different social areas as a kind of special assistant performing rehabilitation tasks due to their structural features $[2,3]$. Especially, the robots which can be used in applications involving dexterous motion skills need natural (smooth) motion patterns while they are performing some complex gestural tasks containing upper/lower torso physical behaviors with arm, hand, finger, leg, neck (head) movements. To acquiring more natural motion patterns in humanoid robots, behaviors observed from biological motor systems which produce new motions reusing and combining existing knowledge before performing the motion in humans and other mammals should be analyzed in detail $[4,5]$.

The fundamental dynamics of the human motor behavior system which is vigorous, versatile, and entirely adaptable present motivation to advanced robotic systems [6-8]. Like the gesturing assignment, the pointing assignment to various targets is one of the typical robotic tasks that human motor skills can be implemented. Besides pointing and gesturing assignments, the manipulation ability of basic tools is an essential asset for these systems.

In conventional systems, movements derived from these tasks should be validated once they are created so that the robot configurations involved are appropriate. Numerous biologically plausible visual and motor coordination models have been developed for achieving these tasks. Eventually, the aftermost objective will be imitating the human mental and motor skills as the fundamental objective is replacing people in perilous and monotonous tasks for improving motor behaviors of robots $[9,10]$. Thus, developing comprehensive computational frameworks for understanding and approximating the motor behavior system of the human brain is one of the sensible approaches to achieve this. As a natural way, computational modeling of the central nervous system and brain can help for developing a novel motion generation and control system for robots [11-13]. 
The cerebellum which may indirectly affect both action and perception is one of the major components in the motor behavior system in the human brain to guarantee dexterous manipulation and coordinated movements although its lesions do not lead to a complete loss of motor functions [14]. Researches promise that it behaves as a forward model giving expected regulatory signals to the motion generation systems processing observed tactile stimuli [15]. Several works including spiking neural networks regard the deviation in taskspace while other studies are dealing with providing the correction margin as an error signal in joint-space. Selforganizing robot navigation in the field with uncertainties has pulled in much consideration of researchers over the previous many years, and numerous biologically plausible heuristic route planning models have been introduced, like the cerebellum and basal ganglia models $[15,16]$. As an approximate model, the cerebellum model which is constructed by information experienced from functional analysis and experiments related to dynamics (or behaviors) of this region in the human brain acts a critical role inside the robust motion generation framework to accomplish accurate and precise movements $[17,18]$. In some examinations, it was shown that the learning cycle depends on the joint space perturbation. Motor control commands transmitted from the spinal cord model of system architecture involve joint angles and torques of the autonomous robot.

In this paper, it is proposed a novel computational neural model to be able to evolve different specific cortical regions of the artificial brain framework which can execute tasks related to cognitive abilities with complex movement-based motor behaviors involving dexterous skills for autonomous robots. Therefore, the framework performing its tasks should continuously adapt to accommodate these changes. This is a difficult issue because of the model complexities. Selforganizing regulators that can handle model vulnerabilities are a reasonable choice for this situation. The computational framework is based on a biologically plausible (spiking) neural model connecting various cortical regions with parallel and distributed network of neural populations which can be attributed spatial-temporal, multi-dimensional, and highdensity information patterns by describing shared or distributed associative memory structures such as long term declarative memories (e.g., semantic and episodic), short term memory (working memory) and procedural memory (behavior motor skills and establishing sensory-motor association functions). As indicated by this viewpoint, a particular issue emerges structure detailing of movement task coordination application which is a basic cognitive skill of artificial brain framework in the robot.

The article following chapter 2 refers to related work which expresses features of previous studies associated with the proposed framework. The following section, chapter 3 determines the design principles of computational braininspired cognitive framework generating, planning, and correcting motor behaviors. Finally, concluding remarks and future works are presented in chapter 4 .

\section{RELATED WORKS}

In the human brain, some areas of cortical and cerebral zones responsible for cognitive functions [19]. Connectionist neural network models such as cellular automata, Boltzman machines, recurrent neural networks, and self-organizing maps comply with the motivation behind the development of computational brain structures that can imitate motor behaviors of humans [20]. To achieve this, various kinds of cells (neurons) may involve in developing the computational structure of a large central nervous system for soft robotic systems [21]. While conventional neural network models utilize the McCulloch-Pitts neuron model, biologically inspired computational neurons such as spiking neural models (e.g, Integrate \& Fire, Izhikevich, and Hodgin-Huxley model) are available (Hodgin et al., 1952) may be preferred in mathematical models of nervous systems for representing human's mental functions [22, 23].

Tieck et al. presented a procedure creating pointing behaviors for a robot with spiking neural model-based architecture [24]. They depicted a basic model of the human engine cortex producing movements utilizing motor functions. The system is trained to perform a simple motor function for pointing at an objective in the middle, and four adjustment functions to point at focuses up, right, left, and down from the base function. According to this, some basic functions can be merged to access various targets. They assess the efficiency of the system embodied a humanoid robot pointing at various targets indicated on a platform. The system achieved to merge one, two, and three motor functions simultaneously to manage the robot progressively to access a particular objective [24]. Besides, they studied to stretch out their work from pointing to a given target to execute a grasping or tool manipulation task. Also, their work has numerous applications for designing and industry including genuine robots. Given the perception and formative component of the science, the study conducted by Wang et al. aims a route planning model utilizing the motivated developmental network (MDN) to emulate the administered learning of the cerebellum and the reinforcement learning dependent on the radial basis function neural network (RBFNN) to mimic the award based learning of the basal ganglia, and coordinates them together to develop a mixture complex comprehension model, to explore a wheeled robot in the area with uncertainty [25]. While the artificial agent is discovering the field for unexplored regions, it employs the cerebellum model to decide action, instead of the greedy method, to speed up the learning optimization performance of the basal ganglia. In addition, their model straightforwardly utilizes the basal ganglia involving the RBFNN to discover and optimize the knowledge base of the cerebellum which allows accomplishing better choice in the accompanying investigation for discovered regions [25]. Their model executes the two-way data transmission between the cerebellum and basal ganglia. Their exploratory outcomes indicate that the model can empower the agent to selfgoverning advancement its knowledge through hybrid learning.

Wu et al. developed a model to imitate visual data processing, motor behaviors and organize peripheral and central neural structures of humans [26]. Their system involves simulating the function of the human dorsal visual pathway ("where" pathway) and the hierarchical structure and function of the human ventral visual pathway ("what" pathway) in humans for localization and recognition of objects respectively [26]. After that, it is responsible for performing motor behavior skills created from the blend of control signals from past experiences, and simulating exact motor actions which imitate calibration of movement errors in past experiences and the alignment of movement from the 
cerebellum to achieve high precision. Their system mirrors designs and elements of human perception (visuomotor coordination), cognition, and accurate motor behavior [26]. Tests on item placement, perception, cognition, and accurate motor behavior exhibit that their model can achieve visuomotor coordination assignments, yet in addition accomplish high exactness development with learning capacity. Then, their outcomes additionally demonstrated the legitimacy of the presented instruments. Besides, their model was implemented to different frameworks, like mechanical and electrical frameworks in mechanical technology, to accomplish quick reaction, high accuracy development with learning capacity.

Zahra et al. studied a work ensuring a completely spiking neural framework that depends on forward prescient learning through a cell cerebellar model [27]. According to their model, the forward model is learned because of the feedback signal over task-space and behaves as a Smith indicator. The last predict perceptual remedies in contribution to a differential matching spiking neural structure while a robot arm controller is experiencing visuomotor coordination behavior [27]. In addition, they improved their control framework to accomplish more precise objective approaching tasks and decrease the movement realization duration for the autonomous systems because of the cerebellar motion prediction abilities. In another study by Zahra et al., a comprehensive cell-based forward cerebellar model involving Golgi and Basket cells was created [28]. To save the bioinspired attributes of the cerebellum in the created model, a hyperparameter optimization strategy updates the weights of the network. The effectiveness of the bio-inspired cerebellar regulator proved for various autonomous systems imitating motor behavior [28].

Kalidindi et al. introduced a cerebellum-based dynamic kinematic regulator. Their dynamic regulator developed on top of a rough reverse kinematic model [29]. In addition, their design ensures mistake minimization without past information about the reverse kinematic regulator and adapts utilizing task space error data [29]. They presented experimental outcomes diminishing tracking error online and the strength of their system. Wilson et al. developed a multizone cerebellar chip for adaptive control of sensorimotor tasks in robots [30]. The multizone cerebellar chip was assessed utilizing a specialized robotic system involving information coming from a group of tactile sensors [30]. They bestowed results of their system with admissible performance for the simultaneous, stable learning in each cerebellar zone. Qiao et al. investigated Brain-inspired intelligent robots which simulate behaviors of humans and animals, from inner mechanisms to external structures, through an integration of visual cognition, decision making, motion control, and musculoskeletal systems [31]. Their review study evaluated the state of the art research in the field of brain-inspired visual cognition, decision making, musculoskeletal robots, motion control, and their integration, which allows robots to perform some complex tasks such as compliant and precise manipulation, fast and flexible response, and deep collaboration between humans and robots [31].

\section{MOTOR BEHAVIOR SYSTEM}

The proposed framework is composed of some different core modules representing cortical regions in the human brain. These modules are the thalamus, basal ganglia, cerebellum, and brainstem. The rest of the other modules including the motor cortex, prefrontal cortex, sensory cortices, spinal cord, and limbic system (amygdala and hippocampus) are considered for supporting this framework as a motion generation system that realizes accurate and precise motor behavior skills. Each module in the computational framework involves a bio-inspired neural model composed of spiking neurons and these modules communicate with each other so that they can realize distributed associative memory structures such as long-term declarative memories (e.g., semantic and episodic), short term memory (working memory) and procedural memory (behavior motor skills and establishing sensory-motor association functions) with spatial-temporal, multi-dimensional and high-density information patterns. Especially, procedural memory known as muscle memory represents network weights in the computational framework to store these information patterns about visual-motor coordination and dexterous motor behaviors for autonomous robots. The motion error evaluated by the cerebellum model in the framework is a key element for motion learning correction as a regulation process over procedural memory like a muscle memory

Thus, as a self-organizing regulator, this framework which can adapt to model vulnerabilities and uncertainties is developed for performing cognitive tasks related to complex movementbased motor behaviors like dexterous skills.

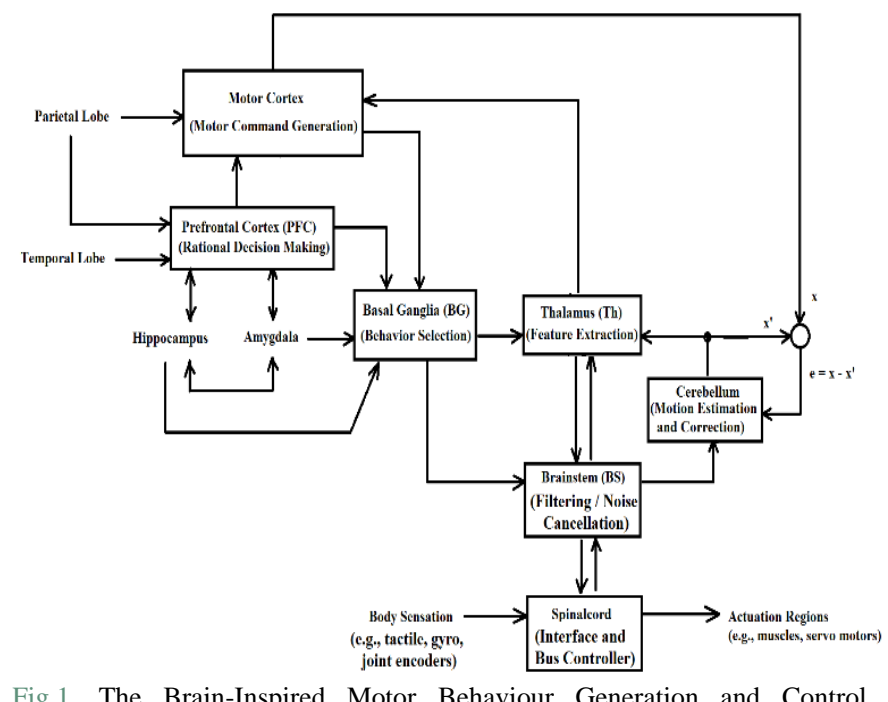

Fig.1. The Brain-Inspired Motor Behaviour Generation and Control Framework

In this computational framework, the basal ganglia model is a critical component that has dense neural links with cerebral cortex regions such as the thalamus, the sensorymotor cortices, the frontal lobe areas (e.g., prefrontal/orbitofrontal cortex), and other brain areas. It contains various nuclei with substantia nigra, striatum, subthalamic nucleus, external and internal globus pallidus.

The basal ganglia model is responsible for many cognitive skills like action selection, motion control, procedural learning related to routine motor behaviors or repetitive (habitual) movements. Mostly, the basal-ganglia model behavior is simulated as the winner-take-all (WTA) principle. According to this, in this computational framework, the basalganglia which is an adaptation mechanism performs a sequential decision-making process over motor commands for motion behavior planning of robots. These tasks executed in the basal-ganglia model are achieved by applying inhibitory 
or excitatory effects on a group of motor systems. While the inhibition signal is suppressing some of the specific motor behavior activities, releasing the excitation signal enables a selected sequence of motor commands (actions) to be activated. The basal-ganglia model in this framework receives information resources from the basal-nucleus (amygdala), subiculum (hippocampus), associative cortices (prefrontal cortex), and motor cortex. Also, it produces response signals to brainstem components (midbrain, medulla, VTA, etc.) and thalamus modules.

The spiking neural network is utilized to build the basalganglia model. The internal topology of the basal-ganglia defining a part of procedural memory is stored in the interconnection matrix as weights. Besides, the basal ganglia model involves reinforcement learning techniques to shape motor behavior sequencing (planning) like switching tasks or action selection by the influence of the inhibitory or excitation signals of the basal ganglia module.

As a model of the computational framework, the brainstem model including some cortical regions like the midbrain, medulla, pons, and ventral tegmental area (VTA) is simply working as filtering, noise cancellation, and dopamine provider system that humanoid robot embodied computational brain inspired cognitive architecture can provide interacting with its dynamic environment. In this model, VTA works as a reward function applied to the basal-ganglia. In addition, a group of nuclei including midbrain, medulla, and pons is considered as an inverse kinematics controller utilizing information with real-time end-effector position.

The computational model of the cerebellum is designed for corrections of movements and motor control [6]. Input stream $u$ (data flow) of the cerebellum model including information related to the desired end-effector motion pattern (displacement trajectory) in task space, and the current changes in each actuator variable (motor command) comes from the brainstem model.

$$
\begin{gathered}
x=\text { cerebellum }(u, e) \\
e=x^{\prime}-x
\end{gathered}
$$

Where $e$ is a correction signal related to the motion error produced by the difference of responses between the cerebellum model $x$ and the motor cortex model $x$ '. It is contributed to coordination, precision, and accuracy of motor control of which timing, positioning (or postural), velocity, and acceleration in the backpropagation algorithm. The computational model of this module acting as an estimator or a non-linear filter can be helped to providing motor learning and improving the quality of movements. The weights are updated by the backpropagation method utilizing the stochastic gradient descent rule.

Like a bridge component, the spinal cord model is a major pathway including multi-modal and multi-channel connections in the central nervous system. Computational aspects of the spinal cord employ some functionalities such as an input-output interface and bus controller module. Also, it is responsible for monitoring and controlling data transmission between brain-inspired cognitive architecture and body regions of the humanoid robot. Body regions in the robot are considered as data acquisition and sensory environment (e.g., tactile sensors, joint encoders, gyro, etc.), actuation environment (e.g., servo motors). To interact the framework with the environment, the spinal cord model receives information from data acquisition and sensory environment (e.g., tactile sensors, joint encoders, gyro, etc.) and it relays motor commands to the actuation environment (e.g., servo motors). The spinal cord module is linked to braininspired cognitive architecture through the brainstem, motor cortex, thalamus models.

In the computational framework, motor commands (actions), or motor behavior primitives which form complex tasks with action sequence are generated by the motor cortex model. Mainly, it receives information from the sensory cortex and thalamus modules. The motor cortex model involves various components such as the primary motor cortex, premotor cortex, supplementary motor area, and posterior parietal cortex [6]. The major aim of the premotor cortex is to perform sensory guidance and direct control of movement. By this region (sub-module), various motor behavior primitives are produced. The primary motor cortex combines these motor behavior primitives to create compound motion patterns. Many different complex behaviors (tasks) can be generated by the sequence of these primitives in the supplementary motor area (SMA). This sub-module behaves as a motion planning engine involving a probabilistic dynamic programming methodology. The mapping process between multisensory information into motor commands is performed by the posterior parietal cortex (PPC). It contains very dense connections with prefrontal regions and sensory regions like visual, auditory, and somatosensory for the perceptual association. In addition, the motor cortex model forms a part of procedural memory related to motor behaviors. Particular motor systems (e.g. body control, arms, legs, and head/face) are modeled as cortical maps in the motor cortex model for neuromorphic robotic systems [7].

\section{CONCLUSIONS}

In this paper, the computational brain-inspired framework related to motor behavior system with cortical modules including the spinal cord, brainstem, thalamus, cerebellum, basal ganglia, and motor cortex is presented via other cortical units such as sensory modules, limbic system (amygdala and hippocampus) and prefrontal cortex. By this paper, as an adaptive control mechanism, a computational neurocognitive framework motivated by the motor behavior system in the human brain is provided. The computational model is mainly constructed by spiking neural structures. This computational framework can be adapted into various types of robotic platforms as well as humanoid robots.

The robot with the framework is efficiently able to simulate task planning problems with multiple targets or motion patterns under predefined cases involving motor behavior tasks. This computational framework is based on the neuromorphic principles of the behavior generation and control mechanism, which is shaped by procedural memory for soft robot systems.

Besides, a reinforcement learning-based adaptation procedure is suitable for behavior sequencing (action selection) procedures in the basal-ganglia model. Motion patterns produced as a result of motor behavior primitives in the motor cortex are constructed by this action sequence process and they are regulated with the cerebellum model.

This proposed framework may be further enhanced in the future, by integrating this framework with central pattern generators. In addition, this brain-inspired architecture can be 
implemented in robotic systems with compliant mechanisms so that different motor behavior functions such as collaborative tasks which are not covered are being realized in this paper.

\section{REFERENCES}

[1] Nagabandi, A., Konolige, K., Levine, S., \& Kumar, V. (2020, May). Deep dynamics models for learning dexterous manipulation. In Conference on Robot Learning (pp. 1101-1112). PMLR.

[2] Arimoto, S. (1995). Fundamental problems of robot control: part II a nonlinear circuit theory towards an understanding of dexterous motions. Robotica, 13(2), 111-122.

[3] Mattar, E. (2013). A survey of bio-inspired robotics hands implementation: New directions in dexterous manipulation. Robotics and Autonomous Systems, 61(5), 517-544.

[4] Ott, C., Eiberger, O., Friedl, W., Bauml, B., Hillenbrand, U., Borst, C., ... \& Hirzinger, G. (2006, December). A humanoid two-arm system for dexterous manipulation. In 2006 6th IEEE-RAS International Conference on Humanoid Robots (pp. 276-283). IEEE.

[5] Maekawa, A., Matsubara, S., Wakisaka, S., Uriu, D., Hiyama, A., \& Inami, M. (2020, April). Dynamic Motor Skill Synthesis with HumanMachine Mutual Actuation. In Proceedings of the $2020 \mathrm{CHI}$ Conference on Human Factors in Computing Systems (pp. 1-12).

[6] Abbatematteo, B., Rosen, E., Tellex, S., \& Konidaris, G. (2021). Bootstrapping Motor Skill Learning with Motion Planning. arXiv preprint arXiv:2101.04736.

[7] Wang, N., Chen, C., \& Di Nuovo, A. (2020). A framework of hybrid force/motion skills learning for robots. IEEE Transactions on Cognitive and Developmental Systems.

[8] Zeng, C., Chen, X., Wang, N., \& Yang, C. (2021). Learning compliant robotic movements based on biomimetic motor adaptation. Robotics and Autonomous Systems, 135, 103668

[9] Liu, R., Zhang, Q., Chen, Y., Wang, J., \& Yang, L. (2020). A Biologically Constrained Cerebellar Model With Reinforcement Learning for Robotic Limb Control. IEEE Access, 8, 222199-222210.

[10] Tolu, S., Capolei, M. C., Vannucci, L., Laschi, C., Falotico, E., \& Hernandez, M. V. (2020). A cerebellum-inspired learning approach for adaptive and anticipatory control. International journal of neural systems, 30(01), 1950028.

[11] Zahra, O., Tolu, S., \& Navarro-Alarcon, D. (2021). Differential mapping spiking neural network for sensor-based robot control. Bioinspiration \& Biomimetics, 16(3), 036008

[12] Shin, D. J. (2020). A Convolutional Neural Network-based Policy Inspired by the Cerebellum.

[13] Yang, S., Wang, J., Zhang, N., Deng, B., Pang, Y., \& Azghadi, M. R. (2021). CerebelluMorphic: Large-Scale Neuromorphic Model and Architecture for Supervised Motor Learning. IEEE Transactions on Neural Networks and Learning Systems.

[14] Parr, T., Limanowski, J., Rawji, V., \& Friston, K. (2021). The computational neurology of movement under active inference. Brain.

[15] Rajendran, A., Vijayan, A., Medini, C., Nair, B., \& Diwakar, S. (2021) Computational modeling of cerebellum granule neuron temporal responses for auditory and visual stimuli. International Journal of Advanced Intelligence Paradigms, 18(3), 356-372.

[16] Zhong, S., Zhou, J., \& Qiao, H. (2021). Bioinspired Gain-Modulated Recurrent Neural Network for Controlling Musculoskeletal Robot. IEEE Transactions on Neural Networks and Learning Systems.

[17] Lobov, S. A., Zharinov, A. I., Makarov, V. A., \& Kazantsev, V. B. (2021). Spatial Memory in a Spiking Neural Network with Robot Embodiment. Sensors, 21(8), 2678.

[18] Azimirad, V., \& Sani, M. F. (2020). Experimental study of reinforcement learning in mobile robots through spiking architecture of thalamo-cortico-thalamic circuitry of mammalian brain. Robotica, 38(9), 1558-1575.

[19] Squire, L., Berg, D., Bloom, F. E., Du Lac, S., Ghosh, A., \& Spitzer, N. C. (Eds.). (2012). Fundamental neuroscience. Academic Press.

[20] Kriegeskorte, N., \& Douglas, P. K. (2018). Cognitive computational neuroscience. Nature neuroscience, 21(9), 1148-1160.

[21] Hassabis, D., Kumaran, D., Summerfield, C., \& Botvinick, M. (2017). Neuroscience-inspired artificial intelligence. Neuron, 95(2), 245-258.

[22] Ghosh-Dastidar, S., \& Adeli, H. (2009). Spiking neural networks. International journal of neural systems, 19(04), 295-308.

[23] Grüning, A., \& Bohte, S. M. (2014, April). Spiking neural networks: Principles and challenges. In ESANN.

[24] Tieck, J., Schnell, T., Kaiser, J., Mauch, F., Roennau, A., \& Dillmann, R. (2019). Generating pointing motions for a humanoid robot by combining motor primitives. Frontiers in neurorobotics, 13, 77.
[25] Wang, D., Hu, Y., \& Ma, T. (2020). Mobile robot navigation with the combination of supervised learning in cerebellum and reward-based learning in basal ganglia. Cognitive Systems Research, 59, 1-14.

[26] Wu, W., Qiao, H., Chen, J., Yin, P., \& Li, Y. (2016). Biologically inspired model simulating visual pathways and cerebellum function in human-Achieving visuomotor coordination and high precision movement with learning ability. arXiv preprint arXiv:1603.02351.

[27] Zahra, O., Navarro-Alarcon, D., \& Tolu, S. (2020). Vision-Based Control for Robots by a Fully Spiking Neural System Relying on Cerebellar Predictive Learning. arXiv preprint arXiv:2011.01641.

[28] Zahra, O., Navarro-Alarcon, D., \& Tolu, S. (2021). A Neurorobotic Embodiment for Exploring the Dynamical Interactions of a Spiking Cerebellar Model and a Robot Arm During Vision-based Manipulation Tasks. arXiv preprint arXiv:2102.01966.

[29] Kalidindi, H. T., Thuruthel, T. G., Laschi, C., \& Falotico, E. (2019, April). Cerebellum-inspired approach for adaptive kinematic control of soft robots. In 2019 2nd IEEE International Conference on Soft Robotics (RoboSoft) (pp. 684-689). IEEE.

[30] Wilson, E. D., Assaf, T., Rossiter, J. M., Dean, P., Porrill, J., Anderson, S. R., \& Pearson, M. J. A Multizone Cerebellar Chip for Bioinspired Adaptive Robot.

[31] Qiao, H., Chen, J., \& Huang, X. (2021). A Survey of Brain-Inspired Intelligent Robots: Integration of Vision, Decision, Motion Control, and Musculoskeletal Systems. IEEE Transactions on Cybernetics.

\section{BIOGRAPHIES}

Evren Daglarli, received the B.Sc. from Marmara University in 2004, the M.Sc. degree in mechatronics engineering with a focus on intelligent systems and robotics from Istanbul Technical University (ITU) in 2007, and the Ph.D. degree in control and automation engineering with a focus on computational cognitive-neuroscience, human_robot interaction from ITU in 2019. He has worked as a Research Assistant with the Department of Electrical and Electronics Engineering, Atilim University. He has many publications that are published in international journals, conferences/symposiums related to mechatronics, intelligent control systems, and robotic areas. As a Researcher, he took some duties and responsibilities in severa national/international projects. He has worked as a Project Engineer and the Department Manager at a private sector technology and engineering company. He is currently working as a Faculty Member and a Lecturer/Instructor with the Computer Engineering Department, Faculty of Computer and Informatics Engineering, Istanbul Technical University. He is a senior member of IEEE. 